\title{
Hydrolyzed eggshell membrane immobilized on phosphorylcholine polymer supplies extracellular matrix environment for human dermal fibroblasts
}

\author{
Eri Ohto-Fujita • Tomohiro Konno • Miho Shimizu • Kazuhiko Ishihara • \\ Toshihiro Sugitate $\cdot$ Jun Miyake $\cdot$ Kotaro Yoshimura $\cdot$ Kaori Taniwaki \\ Takashi Sakurai • Yukio Hasebe · Yoriko Atomi
}

Received: 17 October 2010 /Accepted: 5 April 2011 /Published online: 20 May 2011

(C) The Author(s) 2011. This article is published with open access at Springerlink.com

\begin{abstract}
We have found that a water-soluble alkalinedigested form of eggshell membrane (ASESM) can provide an extracellular matrix (ECM) environment for human dermal fibroblast cells (HDF) in vitro. Avian eggshell membrane (ESM) has a fibrous-meshwork structure and has long been utilized as a Chinese medicine for recovery from burn injuries and wounds in Asian countries. Therefore, ESM is expected to provide an excellent natural material for biomedical use. However, such applications have been hampered by the
\end{abstract}

Eri Ohto-Fujita and Yoriko Atomi contributed equally to this work.

E. Ohto-Fujita $\cdot$ M. Shimizu

Graduate School of Information Science and Technology,

The University of Tokyo,

7-3-1, Hongo, Bunkyo-ku,

Tokyo 113-8656, Japan

T. Konno $\cdot$ K. Ishihara $\cdot$ T. Sugitate $\cdot$ J. Miyake

Department of Bioengineering, School of Engineering,

The University of Tokyo,

7-3-1, Hongo, Bunkyo-ku,

Tokyo 113-8656, Japan

T. Konno $\cdot$ K. Ishihara

Center for NanoBio Integration, The University of Tokyo,

7-3-1, Hongo, Bunkyo-ku,

Tokyo 113-8656, Japan

K. Ishihara

Department of Materials Engineering, School of Engineering,

The University of Tokyo,

7-3-1, Hongo, Bunkyo-ku,

Tokyo 113-8656, Japan

\section{K. Yoshimura}

Department of Plastic Surgery, Graduate School of Medicine,

The University of Tokyo,

7-3-1, Hongo, Bunkyo-ku,

Tokyo 113-8656, Japan insolubility of ESM proteins. We have used a recently developed artificial cell membrane biointerface, 2-methacryloyloxyethyl phosphorylcholine polymer (PMBN) to immobilize ASESM proteins. The surface shows a fibrous structure under the atomic force microscope, and adhesion of HDF to ASESM is ASESM-dose-dependent. Quantitative mRNA analysis has revealed that the expression of type III collagen, matrix metalloproteinase-2, and decorin mRNAs is more than two-fold higher when HDF come into contact with a lower

\section{J. Miyake}

Department of Mechanical Science and Bioengineering,

Graduate School of Engineering Science, University of Osaka,

1-3, Machikaneyama, Toyonaka,

Osaka 560-8531, Japan

K. Taniwaki $\cdot$ Y. Hasebe

Almado incorporation,

2-46-2 Honcho, Nakano-ku,

Tokyo 164-0012, Japan

T. Sakurai

Department of Life Science, Graduate School of Arts \& Science,

The University of Tokyo,

Komaba 3-8-1, Meguro-ku,

Tokyo 153-8902, Japan

Y. Atomi $(\bowtie)$

Radioisotope Center, The University of Tokyo,

2-11-16, Yayoi, Bunkyo-ku,

Tokyo 113-0032, Japan

e-mail: atomi@bio.c.u-tokyo.ac.jp 
dose ASESM proteins immobilized on PMBN surface. A particle-exclusion assay with fixed erythrocytes has visualized secreted water-binding molecules around the cells. Thus, HDF seems to possess an ECM environment on the newly designed PMBN-ASESM surface, and future applications of the ASESM-PMBN system for biomedical use should be of great interest.

Keywords Extracellular matrix $\cdot$ Eggshell membrane . Phospholipid polymer · Dermal fibroblasts · Type III collagen · Human

$\begin{array}{ll}\text { Abbreviations } \\ \text { ESM } & \text { Eggshell membrane } \\ \text { ECM } & \text { Extracellular matrix } \\ \text { ASESM } & \text { Alkaline-digested form of ESM } \\ \text { HDF } & \text { Human dermal fibroblasts } \\ \text { AFM } & \text { Atomic force microscopy } \\ \text { MPC } & \text { 2-Methacryloyloxyethyl phosphorylcholine } \\ \text { MEONP } & p \text {-Nitrophenyloxycarbonyl } \\ & \text { poly(oxyethylene) methacrylate } \\ \text { BMA } & n \text {-Butyl methacrylate } \\ \text { PMBN } & \text { Poly(MPC-co-BMA-co-MEONP) } \\ \text { TC } & \text { Tissue culture } \\ \text { GAPDH } & \text { Glyceraldehyde-3-phosphate dehydrogenase } \\ \text { MMP } & \text { Matrix metalloproteinase } \\ \text { HAS2 } & \text { Hyaluronan synthetase 2 } \\ \text { GAG } & \text { Glycosaminoglycan } \\ \text { PBS } & \text { Phosphate-buffered saline } \\ \text { FGF-2 } & \text { Fibroblast growth factor-2 } \\ \text { TGF- } \beta & \text { Transforming growth factor- } \beta \\ & \end{array}$

\section{Introduction}

Extracellular matrices (ECM) not only serve a structural function providing support and strength to cells within tissues, but also determine critical cellular functions through cellmatrix interactions (Bruckner 2010). The unique architecture and characteristics of tissues and organs are determined by the ECM and the cells that produce it. ECM proteins can be classified into four general categories: collagens, structural glycoproteins, proteoglycans, and elastins (Tsang et al. 2010). In the dermis, heterotypic collagen fibrils containing mainly collagens I, III, and V are the major structural components responsible for its characteristic strength and resilience. Cell adhesion to ECM proteins through physical association with integrins is associated with intracellular signaling events and is critical for successful tissue regeneration (Grzesiak et al. 1997). Mechanical force increases the gene expression for collagen I and III in cycle-stretched cells obtained from ligament, such as anterior cruciate ligament cells (Kim et al. 2002) and bone-marrow-derived mesenchymal stroma cells (Zhang et al. 2008). ECM also plays important functional roles in interacting with numerous growth factors and signaling molecules to regulate cellular events such as cell adhesion, proliferation, migration, survival, and differentiation. Collagens are triple helical proteins that confer compressive and tensile strength to animal tissues and serve as anchors for cell adhesion through surface receptors.

Eggshell membrane (ESM), a functional equivalent of ECM in avian egg during development, is a double-layered insoluble sheet located between the eggshell and egg white and acts as a scaffold for biomineralization to fabricate the egg shell (Rose and Hincke 2009). The membrane is formed in the isthmus of the oviduct of the hen before shell mineralization and egg laying (Rose and Hincke 2009). ESM has a fibrous network mainly comprised of type I, V, and X collagens, glucosamine, desmosin, and hyaluronic acid (Ha et al. 2007; Osuoji 1971; Wong et al. 1984) and is cross-linked by lysyl oxidase (Harris et al. 1980). ESM also has antibacterial and antimicrobial activities to resist bacterial invasion (Ahlborn and Sheldon 2005) and thereby protect the developing embryo (Burley and Vadehra 1989). Recently, ESM-containing cosmetics and supplements have come onto the market worldwide, based on the evidence from traditional folk medicine in Asian countries. For more than four hundred years, ESM has been used to cure injuries, and the prescription appears in the pharmacopoeia of Chinese medicine, Bencao Gangmu. In Japan, Sumo wrestlers use ESM as a natural medicine for injuries. All this evidence suggests that ESM promotes wound healing. However, no molecular mechanism has been studied yet. Wound healing is a four-step sequential event including hemostasis, inflammation, proliferation, and remodeling (Diegelmann and Evans 2004). During these processes, tissue disruption has to be repaired and filled by ECM molecules (e.g., mainly collagen) deposited by dermal fibroblast cells.

In this study, we have aimed to construct a model system for the evaluation of ESM function on dermal fibroblast adhesion and the production of ECM components in vitro. Recently, the construction and modification of biomimetic surfaces has been targeted to support tissue-specific cell functions including adhesion, growth, differentiation, motility, and the expression of tissue-specific genes (von der Mark et al. 2010). We have used a novel artificial cell membrane biointerface, viz., a poly(2-methacryloyloxyethyl phosphorylcholine [MPC]-co- $n$-butyl methacrylate [BMA]-co$p$-nitrophenyloxycarbonyl poly(oxyethylene) methacrylate [MEONP]) (PMBN; Konno et al. 2004) to immobilize alkaline water-miscible organic-solvent-hydrolyzed soluble eggshell membrane (ASESM) molecules. Phosphorylcholine group in the PMBN provides excellent biointerfaces, and these interfaces facilitate the suppression of nonspecific protein adsorption and stabilization of immobilized biomolecules (Watanabe and Ishihara 2007). BMA unit is hydropho- 
bic polymer backbone, which can cover on the surface of substrates such as polystyrene tissue culture dish. MEONP unit contains active ester groups for the conjugation of the amino group of biomolecules via oxyethylene chain (Konno et al. 2004). Although accelerated cell growth of fibroblasts has been observed on an acid oxidized-pepsin digested ESM crosslinked to pepsin-solubilized collagen coat (Ino et al. 2006), our system has a unique advantage for the analysis of the molecular mechanism of ESM-fibroblast interaction. Since cells do not adhere at all to the conventional MPC polymer itself, unlike to collagen-coated dishes or polystyrene cell culture dishes, any specific and direct effects of ASESM molecules on fibroblast adhesion can be tested.

\section{Materials and methods}

\section{ASESM molecules}

ASESM, which is hydrolyzed ESM digested in alkaline water-miscible organic solvent (EM PROTEIN-P), was purchased from Q.P. (Tokyo, Japan).

\section{Microscopic ESM observation}

Avian ESM was removed from eggshell, washed with water, and placed on the slide glass. The surface of the membrane was observed by means of a TCS-SP5 confocal microscope (Leica microsystems, Wetzlar, Germany) with a 488 argon laser.

\section{Atomic force microscopy}

The pyramidal-shaped silicon probe (OMCL-TR400PSA-1, $0.08 \mathrm{~N} / \mathrm{m}$ ) for atomic force microscopy (AFM) was purchased from Olympus (Tokyo, Japan). An MPC polymer such as PMBN was coated onto mica and dried. The mica was placed in a 12-well plate, and then $1 \mathrm{mg} / \mathrm{ml}$ or $30 \mathrm{mg} / \mathrm{ml}$ ASESM was conjugated to it. AFM images of ASESM-conjugated PMBN and non-conjugated control PMBN on mica were taken by using a Nanowizard II (JPK Instruments, Berlin, Germany) in intermittent contact mode in liquid.

Molecular weight estimation of ASESM proteins by size-exclusion chromatography

The molecular weight of ASESM proteins was estimated by size-exclusion chromatography on a Superose 6 HR 10/30 $1.0 \mathrm{~cm} \times 30 \mathrm{~cm}$ size-exclusion column (GE Healthcare, USA) by using high performance liquid chromatography (Waters 640) with the following mobile phase: phosphatebuffered saline (PBS: $137 \mathrm{mM} \mathrm{NaCl}, 8.1 \mathrm{mM} \mathrm{Na} \mathrm{HPO}_{4} \cdot 12$ $\mathrm{H}_{2} \mathrm{O}, 2.68 \mathrm{mM} \mathrm{KCl}, 1.47 \mathrm{mM} \mathrm{KH}_{2} \mathrm{PO}_{4}$ ), at a flow rate of $0.3 \mathrm{ml} / \mathrm{min}$. Thyroglobulin $(670 \mathrm{kDa}), \gamma$-globulin $(158 \mathrm{kDa})$, ovalbumin (44 kDa), myoglobin (17 kDa), and Vitamin $\mathrm{B}_{12}$ $(1.35 \mathrm{kDa})$ in a gel-filtration standard (BioRad) were used to calibrate the column.

\section{Cell culture}

Human dermal fibroblasts (HDF) derived from infant skin were isolated as described previously (Aiba-Kojima et al. 2007). HDF were cultured with Dulbecco's modified Eagle's medium (Wako Pure Chemical Industries) containing $10 \%$ fetal bovine serum (Sigma-Aldrich), $0.1 \mathrm{mg} / \mathrm{ml}$ Kanamycin sulfate (Meiji Seika Kaisha), and a penicillinstreptomycin-neomycin (PSN) antibiotic mixture $(1 \times$; Gibco). HDF of passage number 8 were used in this study.

Synthesis of the PMBN

Methods for preparing PMBN were as previously described by Konno et al. (2004).

\section{Dish coating}

Polystyrene tissue culture (TC) dishes ( $35 \mathrm{~mm}$; Falcon 353001, BD Biosciences) were coated with $0.2 \% \mathrm{PMBN}$, dried in an ethanol atmosphere, coated with $1 \mathrm{ml}$ of various concentrations of $\operatorname{ASESM}(0,1,5,10,30,100 \mathrm{mg} / \mathrm{ml}$ Milli-Q ultrapure water; Millipore) at $4^{\circ} \mathrm{C}$ for $24 \mathrm{~h}$, and then washed with PBS several times. Unreacted active ester units were blocked with $100 \mathrm{mg} /$ $\mathrm{ml}$ glycine at room temperature for $2 \mathrm{~h}$. Before cells were inoculated, the dishes were washed again with PBS. For control non-conjugated PMBN experiments, the active ester units in the PMBN coated surface were inactivated with glycine. PMBN dishes blocked with glycine were prepared. As another control experiment, collagen-coated dishes were prepared as follows: a TC dish was treated with $0.3 \mathrm{mg} / \mathrm{ml}$ collagen (from calf skin; Sigma, C9791) in hydrochloric acid solution (pH 3.0) for $5 \mathrm{~min}$, air-dried, and rinsed with PBS. Cells were observed with an inverted microscope (Zeiss), and images were captured by a charge-coupled device camera (Orca, Hamamatsu, Japan). The brightness and contrast of images were adjusted by Adobe Photoshop Elements v. 6.0.

Quntitative gene expression analysis of HDF with or without ASESM

Total RNA from HDF cultured for $24 \mathrm{~h}$ was extracted by means of the High Pure RNA Isolation Kit (Roche Applied Science, Mannheim, Germany) according to the manufacturer's protocol. First-strand cDNA was synthesized by using PrimeScript RT reagent kit (Perfect Real Time; Takara Bio). The primer and probe oligonucleotides for targeting human genes were designed by utilizing the Universal ProbeLibrary Assay Design Center (Roche Applied Science). All oligonu- 
cleotides were obtained from Nihon gene research laboratories (Sendai, Japan). Amplification of the housekeeping gene glyceraldehyde-3-phosphate dehydrogenase (GAPDH) mRNA, which served as a normalization standard, was carried out with GAPDH primers 5'-AGCCACATCGCTCA GACAC-3' (sense) and 5'-GCCCAATACGACCAAATCC-3' (anti-sense). The gene-specific primers for COL3A1 were $5^{\prime}$ GGACCTCCTGGTGCTATAGGT-3' (sense) and 5'CGGGTCTACCTGATTCTCCAT-3' (anti-sense), for COL1A1 were 5'-GGGATTCCCTGGACCTAAAG-3' (sense) and 5'-GGAACACCTCGCTCTCCA-3' (anti-sense), for matrix metalloproteinase 2 (MMP2) were $5^{\prime}$-GAGG TAATCTTAGGTGCTTACCTAGC-3' (sense) and 5'CTTCAGCACAAACAGGTTGC-3' (anti-sense), for metalloproteinase 3 (MMP3) were 5'-CAAAACATATTTCTTTG TAGAGGACAA-3' (sense) and 5'-TTCAGCTATT TGCTTGGGAAA-3' (anti-sense), for hyaluronan synthetase 2 (HAS2) were 5'-CTCCGGGACCACACAGAC-3' (sense) and 5'-TCAGGATACATAGAAACCTCTCACA-3' (antisense), for elastin were 5'-CACTGGGGTATCCCATCAAG3' (sense) and 5'-GTGGTGTAGGGCAGTCCATAG-3' (antisense), for decorin were 5'-GGAGACTTTAAGAACCT GAAGAACC-3' (sense) and 5'-CGTTCCAACTTCAC CAAAGG-3' (anti-sense), and for biglycan were 5'CAGCCCGCCAACTAGTCA-3' (sense) and 5'-GGCCAG CAGAGACACGAG-3' (anti-sense).

For the quantitative real-time polymerase chain reaction (PCR) analysis, an aliquot of $1 \mu \mathrm{lDNA}$ (25 ng total RNA equivalent) was added to $19 \mu \mathrm{l}$ of the reaction mixture containing $1 \times$ Taqman Universal PCR Master Mix (Applied Biosystems), $900 \mathrm{nM}$ forward/reverse primer, and $250 \mathrm{nM}$ Universal ProbeLibrary probe (Roche). Standard GAPDHbased real-time PCR was performed by using the Applied Biosystems 7500 Fast Real-Time PCR System, and the following PCR cycle was employed: initial denaturation at $95^{\circ} \mathrm{C}$ for $20 \mathrm{~s}$ and then 40 cycles of amplification (denaturing at $95^{\circ} \mathrm{C}$ for $3 \mathrm{~s}$ and annealing and polymerization at $60^{\circ} \mathrm{C}$ for $30 \mathrm{~s}$ ).

To analyze the ASESM dose-dependency of the ECMrelated gene expression pattern, trendlines were calculated and added by Microsoft Office Excel 2007. The best line type was chosen from five different trends (linear approximation, power approximation, exponential approximation, $\log$ approximation, polynomial approximation), so that its $\mathrm{R}$-squared value was at or near 1 .

Immunofluorescence of HDF

Cells were cultured on cover glasses $(22 \mathrm{~mm} \times 22 \mathrm{~mm}, 0.12$ $0.17 \mathrm{~mm}$ thick, Matsunami Glass) in a $35-\mathrm{mm}$ TC dish for $24 \mathrm{~h}$. Cells were briefly washed with PBS and fixed at room temperature in Fix 1 (4\% paraformaldehyde, $2 \mathrm{mM} \mathrm{MgCl}_{2}$, and $2 \mathrm{mM}$ EGTA in PBS) for $10 \mathrm{~min}$. The cells were then washed several times with PBS and permeabilized with Fix2 $(0.03 \%$ Triton $\mathrm{X}-100$ in Fix 1$)$ for $10 \mathrm{~min}$ at room temperature. Fixed cells were washed several times with PBS and blocked in PBS containing 1\% (w/v) bovine serum albumin and $0.02 \%$ sodium azide. Cells were incubated with goat polyclonal anti-procollagen type I antibody (SC-8783, Santa Cruz) overnight at $4{ }^{\circ} \mathrm{C}$, washed with PBS, followed by incubation with DyLight-488-conjugated anti-goat $\operatorname{IgG}$ (H\&L) antibody (Rockland Immunochemicals for Research) for $1 \mathrm{~h}$ at room temperature. Images were taken by using a TCS-SP5 confocal microscope (Leica microsystems, Wetzlar, Germany). The brightness and contrast of images were adjusted by Adobe Photoshop Elements v. 6.0.

\section{Particle-exclusion assay}

To visualize the highly hydrated pericellular matrix around adhered HDF, the fluorescent version of the particle-exclusion assay using fixed sheep erythrocytes was performed. HDF $\left(1.5 \times 10^{4}\right.$ cells/well $)$ was inoculated into 24 -well EZVIEW Glass Bottom Culture Plates LB (ASAHI Glass). After $24 \mathrm{~h}$, WGA (wheat germ agglutinin) Alexa Fluor 488 conjugate (W11261, Molecular probes) at $5 \mathrm{mg} / \mathrm{ml}$ in PBS was added to the culture plate for plasma membrane staining, incubated for $10 \mathrm{~min}$ at $37^{\circ} \mathrm{C}$, and then washed off with PBS. Fixed sheep erythrocytes $\left(5 \times 10^{7}\right.$; Inter-Cell Technologies) were reconstituted and pre-stained with Alexa-488-WGA in PBS, and then excess fluorescent reagent was washed out. Membrane-stained erythrocytes were added to the culture plate, and the cells were visualized with a LSM510 meta confocal microscope (Zeiss).

To estimate the size of the pericellular coat, the distance between the cell outline and the erythrocyte particle was measured (Simpson et al. 2009) by the LSM image browser (Zeiss).

\section{Results}

Physical property of ASESM conjugated to PMBN

The fibril meshwork of the natural avian ESM was observed by confocal microscopy (Fig. 1a). ESM is not a water-soluble material, because it is composed of highly cross-linked ECM molecules. Although commercially available ASESM powder is easy to dissolve to at least $40 \%(\mathrm{w} / \mathrm{v})$ in ultra-pure water at room temperature, undigested and/or re-assembled fibril structure is postulated to remain in the ASESM because of its highly complex fibril architecture (Fig. 1a). The relative molecular weight of ASESM was analyzed by gel filtration, and its main mass was found to be about 12 $14 \mathrm{kDa}$ (Fig. 1b). Newly designed ASESM-PMBN was prepared by covalent bond formation between ASESM 
a

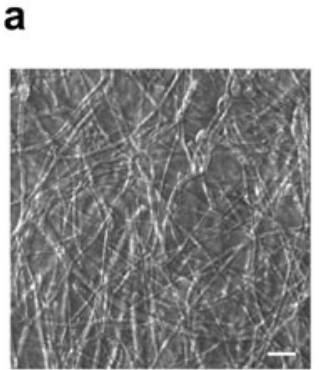

b

C

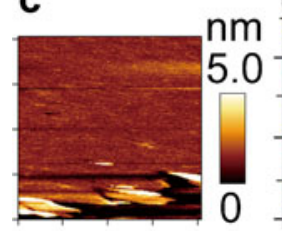

$0_{(\mu \mathrm{m})} 0.4$

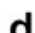

d

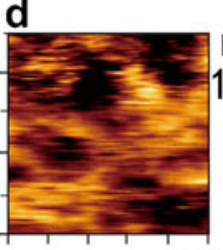

0.5
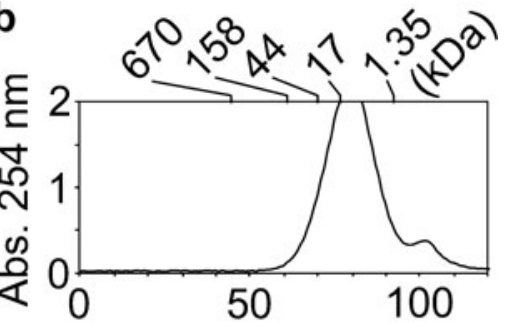

Time (min)

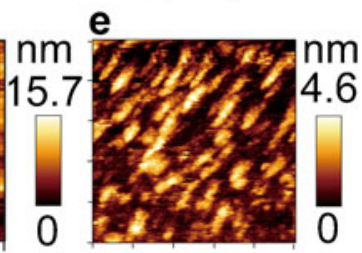

0

0.5

$(\mu \mathrm{m})$

Fig. 1 a Microscopical image of eggshell membrane (ESM). Bar $100 \mu \mathrm{m}$. b Distribution of molecular weight of alkaline water-miscible organic-solvent-hydrolyzed soluble eggshell membrane (ASESM) molecules as estimated by size-exclusion chromatography. Five molecular weight protein standards are indicated above the chart corresponding to the respective elution time. c-e Fibril structures forming when $30 \mathrm{mg}$ / $\mathrm{ml}$ ASESM is conjugated to PMBN [poly(2-methacryloyloxyethyl phosphorylcholine- $c o-n$-butyl methacrylate- $c o-p$-nitrophenyloxycarbonyl poly(oxyethylene) methacrylate)]. AFM images when $0 \mathrm{mg} / \mathrm{ml}$ (c), $1 \mathrm{mg} / \mathrm{ml}$ (d), or $30 \mathrm{mg} / \mathrm{ml}$ (e) ASESM is conjugated to PMBN on mica. Full color range corresponds to a vertical scale of 4.6-15.7 nm

proteins and PMBN, which has active ester groups (Konno et al. 2004). AFM analysis of ASESM-PMBN applied to mica showed a distinct fiber-like structure when $30 \mathrm{mg} / \mathrm{ml}$ ASESM was used for PMBN conjugation (Fig. 1e) but at not the lower concentration of ASESM (1 mg/ml; Fig. 1d). As a control, PMBN alone did not give any detectable structure (Fig. 1c). The typical 67-nm banding pattern of collagen fibrils was not observed in ASESM-PMBN, which is consistent with the result for ESM reported by Wong et al. (1984). ESM is a double-layered fibrous membrane consisting of cross-linked ECM proteins and is formed by lysyl oxidase secreted by the cells inside the hen oviduct during egg formation (Harris et al. 1980). A detailed structural analysis of ASESM fibrils on PMBN will be interesting, because this newly prepared biointerface provides a natural nano-scale fibrous scaffold for HDF as described below.

\section{Adhesion of HDF to ASESM conjugated to PMBN}

HDF adhesion to ASESM was initially tested in a plane TC dish. Either with or without ASESM pre-coating, cells adhered to the dish with no detectable difference (data not shown). To obtain a dish surface to which HDF adhered only when ASESM was present, a cytiocompatible MPC polymer bearing active ester units, PMBN (Konno et al. 2004) was used, which can immobilize biomolecules (e.g., proteins,

polysaccharides, and DNA) via covalent bond formation between the amino groups of target biomolecules and active ester units in the polymer under mild conditions. First, PMBN was coated onto a TC dish, and then various concentrations of ASESM $(0,1,5,10,30,100 \mathrm{mg} / \mathrm{ml})$ were applied for conjugation (Fig. 2a-f). Non-reacted active ester groups in the PMBN were blocked by incubation with glycine, and then the dishes were washed with PBS before $8 \times 10^{4}$ HDF were seeded into them. After $24 \mathrm{~h}$, cells were observed under the inverted microscope. We found that HDF had adhered to the ASESM-PMBN dishes (Fig. 2b-f) specifically, as compared with control glycine-conjugated PMBN dishes without ASESM (Fig. 2a). When more than $30 \mathrm{mg} / \mathrm{ml} \mathrm{ASESM} \mathrm{was} \mathrm{used} \mathrm{for} \mathrm{conjugation} \mathrm{with} \mathrm{the}$ PMBN, the cell culture became semiconfluent (Fig. 2e, f), as in the dishes coated with type I collagen (Fig. $2 \mathrm{~g}$ ) and in untreated TC dishes (Fig. 2h). Interestingly, the mode of cell adhesion was ASESM dose-dependent when used with PMBN conjugation (Fig. 2b-f), but not with respect to the number of the cells inoculated. Notably, cells were relatively round and seemed not to be fully attached to the matrix formed by the lower does of ASESM $(1 \mathrm{mg}, 5 \mathrm{mg} / \mathrm{ml}$ ASESM-PMBN in Fig. 2b, c, i). However, on the ASESM scaffold in which higher doses of ASESM were conjugated to the PMBN, the cells were extended and flat (Fig. 2f, k, n, p), similar to the cells on control TC/collagen-coated dishes (Fig. 2h, 1, m, o), and fully adhered to the matrix regardless of the cell density on the ASESM surface (adjusted by number of cells seeded). The morphology of HDF on ASESM at $10 \mathrm{mg} / \mathrm{ml}$ (Fig. 2j) showed an intermediate structure between the cells on ASESM at $5 \mathrm{mg} / \mathrm{ml}$ (Fig. 2i) and ASESM at $100 \mathrm{mg} / \mathrm{ml}$ (Fig. 2k) /control TC dish (Fig. 21). These different cell adhesion modes, which various doses of ASESM were used for PMBN conjugation, may reflect the surface structure (fibril plus or minus) as observed in AFM analysis (Fig. 1d, e).

\section{ECM-related gene expression of HDF on ASESM conjugated to PMBN}

Expression of the major ECM-related genes from HDF attached to different doses of the ASESM-conjugated matrix was examined by a standard quantitative real-time PCR protocol, and the gene expression pattern as a function of ASESM dose was analyzed by a curve fitting method (Fig. 3). The gene expression pattern of type III collagen (Fig. 3a), decorin (Fig. 3g), and MMP2 (also known as gelatinase A, Fig. 3e) at the various doses of ASESM was fitted with a power approximation curve, unlike the other genes: type I collagen (Fig. 3b), elastin (Fig. 3c), MMP3 (Fig. 3d), biglycan (Fig. 3h), and HAS2 (Fig. 3f). Interestingly, at the lower doses of ASESM conjugated to the MPC surface $(1 \mathrm{mg} / \mathrm{ml}$ ASESM, the leftmost graph in each individual gene 
Fig. 2 HDF adheres preferentially to ASESM fibril structure, and the mode of HDF adhesion is controlled by the ASESM dose, which affects the fibril structure on PMBN, but not by the population of the cells. ah HDF at $8 \times 10^{4}$ cells/dish were grown on $0 \mathrm{mg} / \mathrm{ml}$ (a), $1 \mathrm{mg} / \mathrm{ml}$ (b), $5 \mathrm{mg} / \mathrm{ml}$ (c), $10 \mathrm{mg} / \mathrm{ml} \mathrm{(d),}$ $30 \mathrm{mg} / \mathrm{ml}$ (e), or $100 \mathrm{mg} / \mathrm{ml}$ (f) ASESM conjugated to PMBN as a matrix, on a collagen matrix (g), or on a control (directly on tissue culture [TC] dish) surface (h). Bars $100 \mu \mathrm{m}$. i, j Representative cells in c, $\mathbf{d}$ (arrowheads), respectively, at higher magnification. k, I Dotted boxes with arrowhead in $\mathbf{f}, \mathbf{h}$, respectively, at higher magnification. Bars $20 \mu \mathrm{m}$. m, n HDF at $1 \times 10^{3}$ cells/dish were grown on a control collagen matrix) or on $100 \mathrm{mg} / \mathrm{ml}$ ASESM conjugated to PMBN, respectively. Bars $100 \mu \mathrm{m}$. o, p Representative cells in $\mathbf{m}, \mathbf{n}$ (arrowheads), respectively, at higher magnification. Bars $20 \mu \mathrm{m}$
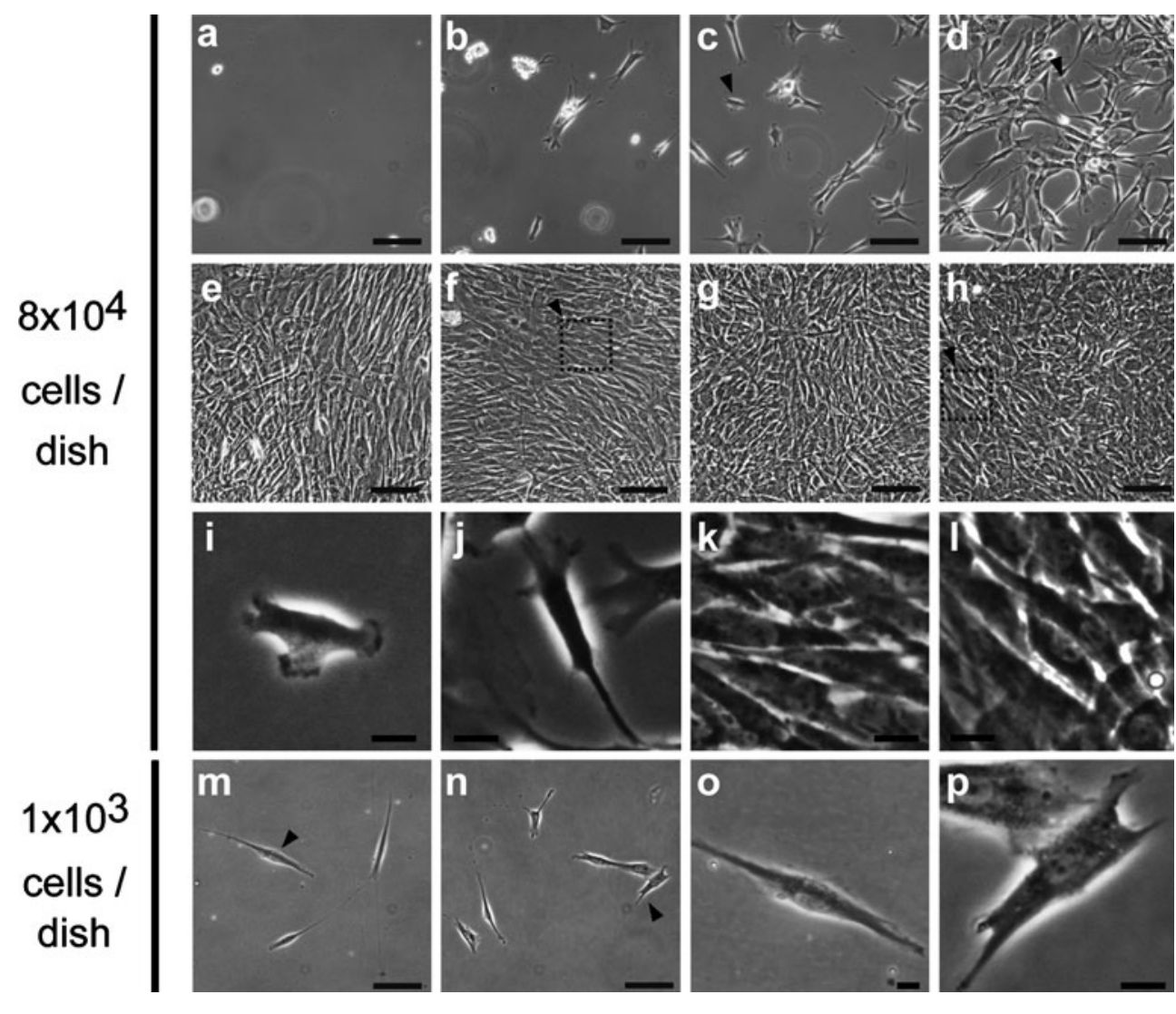

set in Fig. 3), the mRNA expression level of type III (Fig. 3a) but not type I collagen (Fig. 3b), MMP2 (Fig. 3e), and decorin (one of the proteoglycans that contains one chondroitin/dermatan sulfate GAG side-chain, Fig. 3g) was markedly increased by more than two-fold compared with control TC or collagen-coated dishes (Fig. 3a, e, g, inset). Other ECM-related genes such as type I collagen (Fig. 3b), elastin (Fig. 3c), MMP3/stromelysin-1 (Fig. 3d), and biglycan (Fig. 3h) showed similar mRNA expression levels as the control, with no ASESM dose-dependency. Expression of another ECM constituent, HAS2, which has a high molecular weight with unbranched polysaccharide extruded to the extracellular space, increased about three-fold depending on ASESM dose but was the same level as control (Fig. 3f). From these mRNA expression analyses, type III collagen, MMP2, HAS2, and decorin were found to be direct/indirectresponse genes of HDF by ASESM proteins. Further study (e.g,. time-course of gene and protein expression and identification of the related growth factors/cell signaling mechanism) will clarify the ASESM/ESM function toward HDF.

Visualization of ECM proteins and glycosaminoglycan in HDF: type I collagen and pericellular ECM

We also analyzed the ECM-related gene expression at the cellular level with or without ASESM in HDF by antibody detection of type I collagen and by particle-exclusion assay for detecting the highly hydrated ECM at the pericellular space (Fig. 4). HDF were grown on an ASESM surface (Fig. 4c, d), on a surface coated with type I collagen (Fig. 4b), or on glass (Fig. 4a) and fixed. Indirect immunofluorescence detection of procollagen type I was performed. The antibody recognizes the procollagen in the endoplasmic reticulum. The localization pattern was similar between the cells on the type I collagen coat (Fig. 4b) and on higher doses of the ASESM conjugated surface (Fig. 4c, d) consistent with the result as shown in Fig. 2o, p.

ECM-related glycosaminoglycan (GAG), such as decorin and hyaluronan (gene product of HAS2), is responsible for various cellular function including adhesion and wound healing (Laurent and Fraser 1992). GAG is a highly hydrophilic molecule that exists on the cell surface and binds a large amount of water around the cell. Visualization of such a pericellular coat can be achieved by the reported particle-exclusion assays on fixed erythrocytes for hyaluronan detection (Itano et al. 1999; Fig. 4e-h). The distance from the cell surface to the excluded area is similar between low and high doses of ASESM. Although the HAS2 expression was relatively low for HDF on lower dose of ASESM surface (Fig. 3f), HDF appeared to secrete similar amounts of pericellular coat as on other culture surfaces. A relatively clear exclusion pattern was seen for the cells on a lower dose of ASESM surface (Fig. 4f). This might be correlated to the higher decorin mRNA expression (Fig. 3g), 
Fig. 3 Relative gene expressions of HDF on various amounts of $\operatorname{ASESM}(1,5,10,30,100 \mathrm{mg} / \mathrm{ml})$ conjugated to PMBN as normalized with glyceraldehyde-3phosphate dehydrogenase: type III collagen (COL3; a), COL1 (b), elastin (c), matrix metalloproteinase (MMP3; d), MMP2 (e), hyaluronan synthetase 2 (f), decorin (g), and biglycan (h).

The assay was carried out on triplicate samples with $500 \mathrm{ng}$ total RNA recovered from each dish. The data are presented as means \pm SD (arb. units arbitrary units). The fitted curves are as described. Insets Relative gene expressions in HDF on either dishes coated with type I collagen $(C O L)$ or untreated tissue culture dishes (TC). Horizontal axis in each graph represents various concentrations of ASESM conjugated to PMBN (mg/ml)
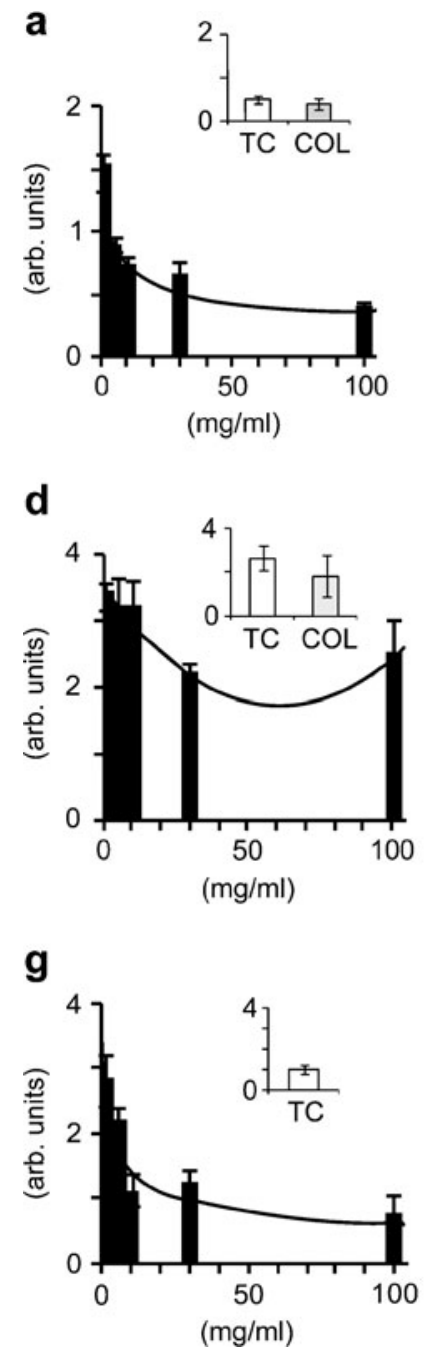
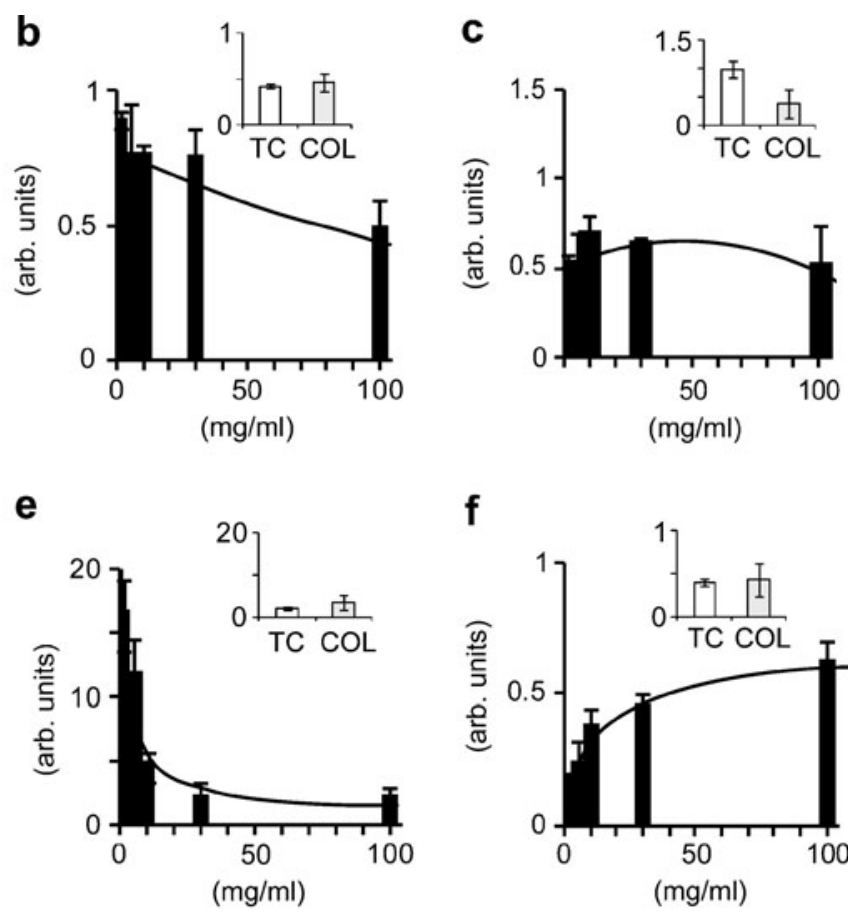

h
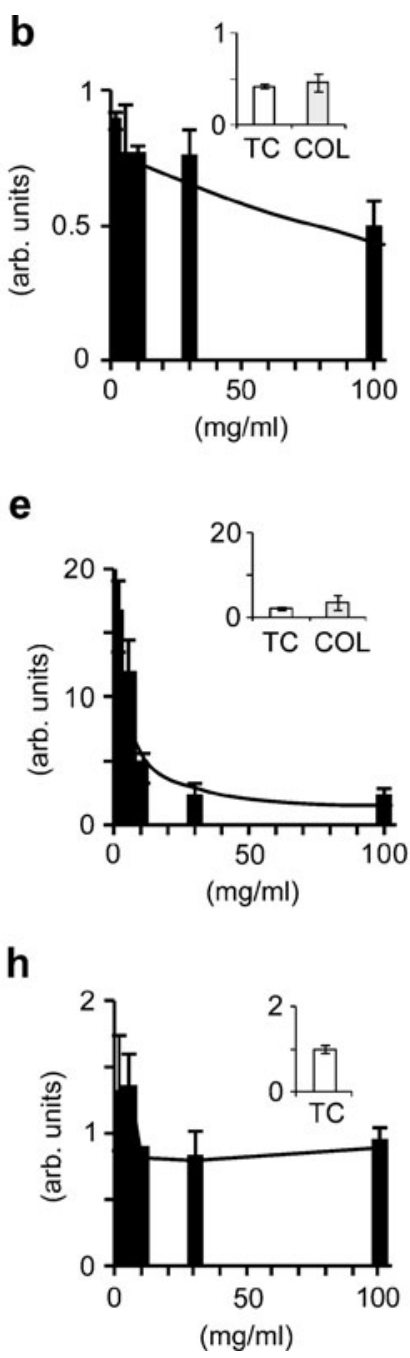
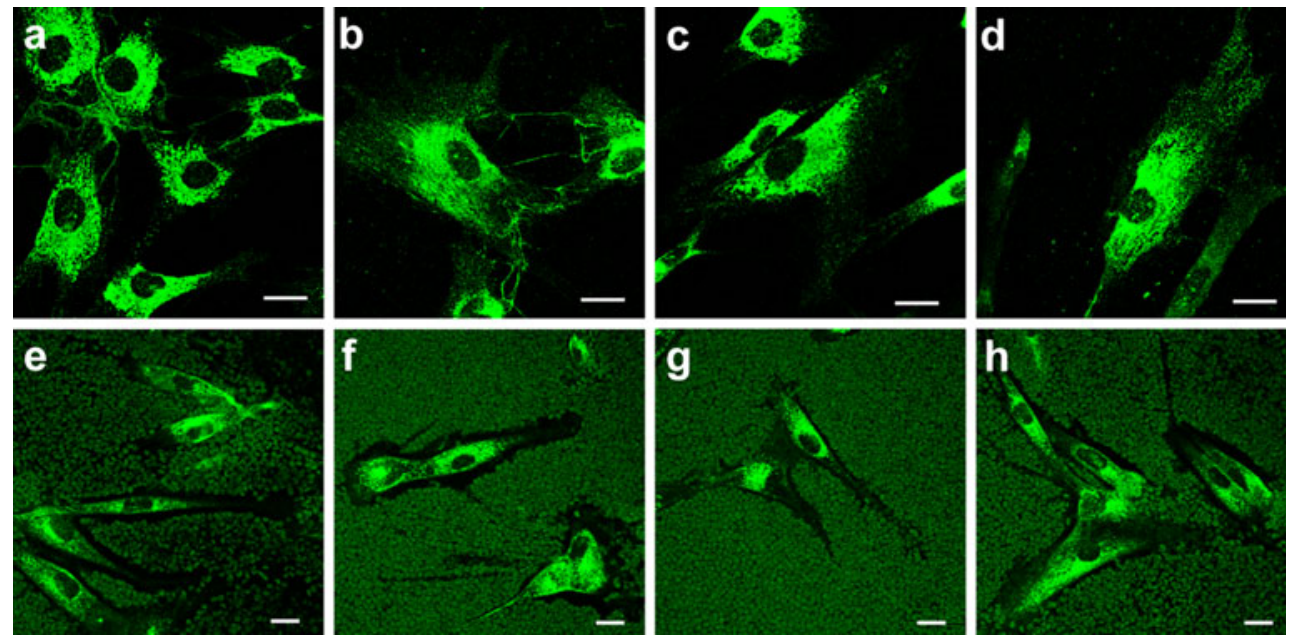

Fig. 4 Visualization of type I collagen protein and particle-exclusion assay on HDF. Cells were grown on glass (a), a collagen coat (b), $30 \mathrm{mg} / \mathrm{ml} \mathrm{ASESM-PMBN}$ coat (c), or $100 \mathrm{mg} / \mathrm{ml} \mathrm{ASESM-PMBN}$ coat (d). Bar $20 \mu \mathrm{m}$. Highly hydrated ECM-related glycosaminoglycan coat around HDF grown on glass (e), or on $1 \mathrm{mg} / \mathrm{ml}$ (f), $10 \mathrm{mg} / \mathrm{ml}$ (g), or $100 \mathrm{mg} / \mathrm{ml}$ (h) ASESM-PMBN coat. Bars $20 \mu \mathrm{m}$ 
and/or the distinct motility of the cells (Evanko et al. 1999) might responsible for this observation as previously described in Fig. 3. The distance from cell surface to excluded area is not different between low and high doses of ASESM. These data show that HDF on the ASESM surface secrete a pericellular coat, including hyaluronan, as on other culture surfaces.

\section{Discussion}

We have developed a new in vitro system to study ESM function with respect to HDF adhesion by applying the PMBN. ESM is a readily available natural biomaterial everywhere in the world and can be used in the biomedical, nutritional, and cosmetic fields. The membrane is a highly cross-linked meshwork mainly consisting of ECM molecules such as collagens, proteoglycans, and GAG. A commercially available, alkaline-hydrolyzed version of ESM, ASESM, has been used in this study. Based on AFM analysis, ASESM has been found to adopt a distinct fibril structure compared with the original ESM, either because hydrolysis-tolerating fibers remain and/or hydrolyzed ECM are reassembled de novo on PMBN. An analysis of this assembly process will be interesting from two different viewpoints: (1) in order to understand the HDF adhesion mode and to develop a new biointerface by using ESM and thereby providing different cell adhesion and gene expression properties, and (2) in order to understand an as yet unknown eggshell/shell membrane formation mechanism inside the oviduct of the hen, a mechanism expected to provide hints for designing new biomaterials.

In this study, specific dish surface ASESM conjugated to PMBN was prepared and tested for HDF adhesion. Cells adhere to ASESM on PMBN specifically (Fig. 5b, c) but not to glycine-blocked PMBN (Fig. 5a), and ECM genes are expressed. The most notable finding from our study is that ECM gene expression is significantly altered simply by the cell density and microenvironment of the cells and suggests the following two important points: first, the specific dish surface of ASESM conjugated to PMBN provides a useful in vitro experimental culture system mimicking the actual cell environment inside our living human body; second, the present study provides the first mechanistic insight into the reason that ESM encourages scarless wound healing, as has been known for many years in Asian countries.

Fibroblasts are generally and widely used cultured cells in the field of biological sciences and almost all reported experiments are performed under confluent cell conditions with no thoughts of the in vivo situation. If we look at any of the histological images from tissue sections or the in vivo images of dermis, tendon, and joints in our body, we can readily notice that fibroblasts are dispersed and not "confluent". In spite of the importance of ECM, few studies have investigated tissue origin-dependent cell-ECM interactions or cell-cell interactions in vivo. To visualize such interactions, a histological approach is commonly used, but the dynamic response of the cells to the environments and the modulation of the cytoskeleton and ECM cannot be studied. In the case of recently developed induced pluripotent stem cells (iPS; Takahashi and Yamanaka 2006), robust and intensive studies have been performed world-wide, and a quick shift from basic research to practical applications has been brought about, because such investigations are based on an easily controllable, in vitro cell culture technology. With in vitro systems, cells that survive in various regions of the body (tissue) can be analyzed not only under various nutritional factor conditions, but also with regard to the stiffness of their substrate (Discher et al. 2005). Three-dimensional culture systems are certainly more preferable than two-dimensional systems, because

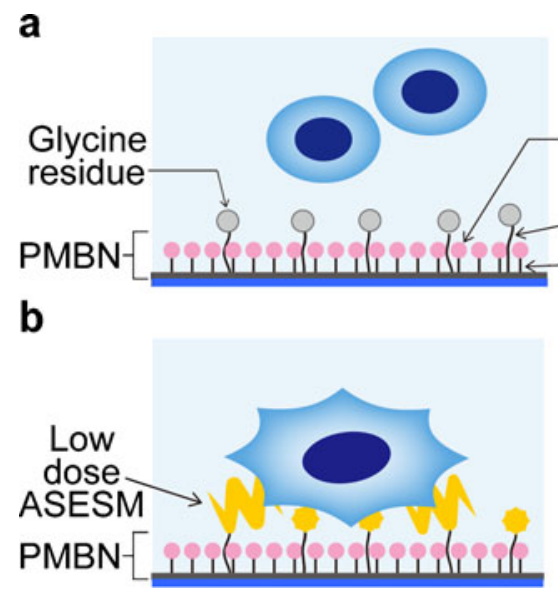

Fig. 5 Characteristic features of the ASESM-PMBN system. a Without ASESM, cells do not adhere to PMBN, because unreacted active ester groups were blocked with glycine. b Low dose of
Phosphorylcholine group Oxyethylene chain Hydrophobic polymer backbone

C

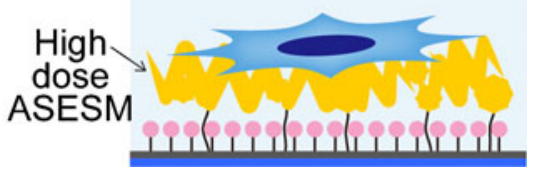

ASESM conjugated to PMBN provides early wound healing model. c High dose of ASESM conjugated to PMBN provides a more stretched and rigid environment 
they more closely resemble in vivo conditions, and the mechanical character of ECM and the change of gene expression induced by an environmental cue can be detected (von der Mark et al. 2010). However, complex three-dimensional systems are not always necessary for the direct analysis of the cells responding to a specific biomaterial, as described in this study. Gene expression supplies the ECM environment to HDF, and this may partially explain the biomedical function of ESM for the healing of skin injuries (wound healing). Indeed, Rinn et al. (2008) have demonstrated that dermal fibroblasts are developmentally regulated by particular HOX genes dependent on the different regions in the body. Fibroblasts originating from other skin regions of the body or connective tissues might respond to ASESM differently from those taken from the auricle as used in this study. Our newly developed experimental system might be suitable for investigating site-specific cell response in general. Further studies should clarify the complex biological mechanism regarding the association of HDF and other adhesive cells to ASESM, by adding/subtracting the involved factors in a step-by-step fashion.

We have studied mRNA expression by the quantitative real-time PCR analysis of HDF grown on ASESM. ECMrelated genes, which might contribute to maintaining the extracellular environment for healthy dermis, such as those for type III collagen (Vitellaro-Zuccarello et al. 1992), MMP2 (Kahari and Saarialho-Kere 1997), and decorin (Nomura 2006), show a more than two-fold higher expression when cells are grown at a lower dose of ASESM on a surface than when they are cultured on a control dish surface. In the contrast, HAS2 expression is high at a higher dose of ASESM on the surface.

Cutaneous wound healing is a dynamic process that involves the coordinated and sequential deposition of ECM, leading to the formation of a scar. In this study, during the culture of HDF on ASESM for just $24 \mathrm{~h}$, an interesting pattern of gene expression of ECM components including collagen, proteoglycans and hyaluronan and their proteases has been observed, as a function of the applied ASESM quantity. This ASESM dose-dependency seems to be consistent with the temporal expression pattern of ECM molecules during sequential wound healing process. The ECM expression pattern in low-dose ASESM is similar to the early phase, whereas that at a high dose resembles the late phase of wound healing. To analyze the ASESM dosedependency of ECM-related gene expression patterns, a trendline can be added to the graph by a curve fitting method (Fig. 3). Type III collagen, decorin, and MMP2 are strongly expressed in the early phase of wound healing. In our study, the expression pattern of these three genes have been fitted into a downward power approximation toward the high ASESM dose, whereas other genes have been fitted by the binomial equation, exponential equation, or log approximation. Interestingly, the ASESM dose-dependent increment of HAS2 mRNA fits an upward exponential equation. Although we have not checked the gene expression at a time point other than $24 \mathrm{~h}$, the level of mRNA expression of these wound-healing-related genes at different ASESM concentrations matches the gene expression during the course of sequential events of cutaneous wound healing in vivo; lower ASESM providing an environment similar to the early steps, and higher ASESM for late steps. Based on our AFM analysis (Fig. 1c-e), ASESM seems to adopt a distinct fibril structure depending on the concentration of ASESM applied to PMBN for conjugation, either because the hydrolysis-tolerating fibers remain and/or hydrolyzed ECM are reassembled de novo on PMBN.

Since mutations for both type III (Liu et al. 1997) collagen and decorin (Jarvelainen et al. 2006) are not lethal in the mouse, these genes are not essential for survival but are important for providing a soft environment within tissues and play roles in modulating the ECM environment, being responsible for elastic young skin, for human health, and for body maintenance in aged people. The major function of type III collagen is associated with the fibrogenesis of type I collagen (Liu et al. 1997). Type III collagen is a fibrillar forming collagen comprising three alpha-1 (III) chains and is expressed in early embryos and throughout embryogenesis. In the adult, type III collagen is a component of the ECM in a variety of internal organs and skin. Patients with type IV Ehlers-Danlos syndrome, a genetic disorder associated with fragile blood vessels and skin, often carry mutations in the COL3A1 gene encoding for type III procollagen (Kontusaari et al. 1990; Kuivaniemi et al. 1995; Prockop and Kivirikko 1984). Type III collagen is important for the development of skin and the cardiovascular system and for maintaining the normal physiological functions of these organs in adult life (Olsen 1995). In addition, type III collagen is known to be prominent at sites of healing and repair in skin and other tissues (Wu et al. 2010). Type III collagen is the major constituent of early granulation and scar tissue, whereas only a small amount of type I collagen is present in this early phase of healing (Betz et al. 1993; El Sherif et al. 2006). On the other hand, type I collagen is a major fibrous component in connective tissue. During the wound healing process, type I collagen accumulates in granulation tissue (Kanzler et al. 1986). Because the expression of type III collagen is usually greater than the expression of type I collagen during early wound healing, the lower ASESM-PMBN in vitro system might provide an ECM environment similar to that of the early phase of wound healing.

Decorin belongs to the small leucine-rich proteoglycans (SLRPs), which are expected to play important functions in tissue assembly. Danielson et al.(1997) have reported that mice possessing a targeted disruption of the decorin gene 
are viable but have fragile skin with markedly reduced tensile strength. Since the repair of wound healing is delayed in the decorin-knockout mouse, decorin proteogrican is necessary for the wound healing process (Jarvelainen et al. 2006). In keloid scars seen in deregulated wound healing, decorin expression is downregulated, whereas syndecan-2 and fibroblast growth factor-2 (FGF-2) are upregulated (Mukhopadhyay et al. 2010). In addition, the activation of extracellular signal-regulated kinase-1 (ERK1) and ERK2 and the lower expression of decorin are found in the fibroblasts of the dermal region in keloids (Meenakshi et al. 2009). On the other hand, the overexpression of the decorin gene in human corneal fibroblasts inhibits transforming growth factor- $\beta$ (TGF- $\beta$ )-induced fibrosis and myofibroblast formation (Mohan et al. 2010). These reports suggest that decorin has an antifibrotic effect in an aberrant wound healing process leading to keloid scars.

MMPs play a role in various aspects of cutaneous biology and pathology, e.g., in wound repair. MMP2 is expressed in the early phase (7th day) of wound healing (Jansen et al. 2007). MMP2 expression is high in low-dose ASESM in our in vitro system. MMP2 degrades various ECM molecules, such as collagen types I, IV, V, VII, and $\mathrm{X}$, gelatin, fibronectin, tenascin, fibrillin, osteonectin, entactin, aggrecan, vitronectin, and decorin (Kerkela and Saarialho-Kere 2003) and is activated by dermatan sulfate (Isnard et al. 2003). Since dermatan sulfate is reported to be one of the components of ESM (Osuoji 1971), it is also present in our ASESM system and might contributed to MMP2 activation. MMP2 seems to be a key molecule for tissue remodeling, as demonstrated by Tholozan et al. (2007) who have shown that lens epithelial cells produce several MMPs including MMP2, which releases FGF-2 from the lens capsule. In this system, the recently proposed ECM reservoir hypothesis has been established, because the ECM itself can act as a reservoir for growth and survival factors that are released via the action of various MMPs such as MMP2 (Tholozan et al. 2007). Thus, lower density cell culture on low ASESM might provide a good environment for wound healing without leading to keloid scar formation during tissue regeneration.

In vivo, fibroblast cells are located separated from each other, which differs from the situation for keratinocytes in the epidermis. As described above, collagen type III, decorin, and MMP2 play an important role in wound healing, type I collagen fibrogenesis, and the maintenance and turnover of the ECM environment during skin damage. In this study, sparsely located HDF show an unextendedshape on a low-dose ASESM matrix, whereas the mRNA expression levels of type III collagen, MMP2, and decorin markedly increase more than two-fold compared with those of cells grown on control TC or collagen-coated dishes. The proteins encoded by these ECM genes are key molecules for remodeling of ECM in wounded and developing dermis and contribute to keeping the skin healthy. However, the precise mechanisms of their involvement are largely unknown.

HDF express collagen type III, MMP2, and decorin mRNA at a higher level on a low dose of ASESM, but the levels of collagen type I, elastin, MMP3, and biglycan are not significantly altered. Biglycan belongs to the group of small leucine-rich proteoglycans, as does decorin, and decorates collagen during fibrogenesis (Corsi et al. 2002). Decorin and biglycan expression patterns are reported to be differentially regulated during wound healing. In dermal fibroblast cultures, decorin expression is stimulated by glycyl-histidyl-lysine- $\mathrm{Cu}^{2+}$, which is a tripeptide isolated from human plasma and a potent activator of wound healing. In contrast, biglycan expression is not modified (Pickart and Thaler 1973). In addition, the inactivation of the decorin gene by homologous recombination (Danielson et al. 1997; Xu et al. 1998), but not that of biglycan (Simeon et al. 2000), is associated with a fragile skin collagen network. A previous study has also revealed few changes occur with regard to biglycan expression through 1-49 days of the wound healing process in pig (Wang et al. 2000). Thus, we can reasonably conclude that different regulatory mechanism are also involved in the patterns of expression of decorin and biglycan in our system, despite both belonging to the small leucine-rich proteoglycans.

Elastin, which constitutes elastic fibers in the dermis, blood vessel, and lung (Rosenbloom et al. 1993), did not change its expression level related to ASESM quantity in this study. However, a relatively low expression level was observed under lower dose ASESM conditions. A previous study has indicated that elastin expression increases during the early phase of wound healing (Quaglino et al. 1990). Basic fibroblastic growth factor has been reported to be of importance in the regulation of ECM gene expression, such as the decrease of elastin expression (Debelle and Tamburro 1999) but increase of type III collagen and decorin (Li et al. 2009; Tan et al. 1993). Although growth factors/cytokines were not measured in this study, the dose-dependent change of ECM mRNA may well reflect the ECM-cytokine relationship during wound healing described in previous reports.

The expression pattern of MMP3 mRNA at the different ASESM concentrations are a mirror image of the pattern of elastin expression. MMP3 is secreted mainly by macrophages and fibroblast cells (Saarialho-Kere et al. 1994; Welgus et al. 1990; Wilhelm et al. 1987) and digest a broad range of ECM molecules, such as type I collagen, various proteoglycans, and others (ColIII, IV, V, VII, IX, and X, elastin, fibronectin, fibrillin, fibrinogen, gelatin, aggrecan, plasmin, kallikrein, chymase,LN-1, nidogen, vitronectin, osteonectin, decorin; Kerkela and Saarialho-Kere 2003), as substrates. The higher MMP3 mRNA expression either at 
low ASESM (ASESM at 1, 5, $10 \mathrm{mg} / \mathrm{ml}$ ) or high ASESM (ASESM at $100 \mathrm{mg} / \mathrm{ml}$ ) might indicate that ECM turnover is high under these conditions. Delayed and incomplete wound healing is observed in the MMP3-knockout mouse resulting from insufficient myofibroblast migration or differentiation. Therefore, MMP3 function is important for proper wound healing, and our ASESM system provides the necessary environment.

Interestingly, a large difference has been noted in HDF cell shape between low- and high-dose ASESM (Figs. 2, 5b, c), suggesting that different actin dynamics and related signal transduction mechanisms are involved in the two states. Hyaluronan expression level seems to change in relation to the cellular and extracellular metabolism and to the immune system through actin dynamics (Boraldi et al. 2003; Stern 2003). Particle-exclusion assay in this study shows waterbinding ECM surrounding HDF cells grwon on both low and high dose ASESM. The distance of the water-binding ECM from the cell surface to the excluded erythrocyte is similar between low and high ASESM. This data suggest that HDF on the ASESM surface secretes enough pericellular coat, including GAG-bound proteoglycan and hyaluronan, at a low dose of ASESM and at a high dose of ASESM. The pericellular hyaluronan coat requires TGF- $\beta$ expression (Simpson et al. 2009). The differentiation of fibroblasts to myofibroblasts during wound healing needs the expression of epidermal growth factor receptor and ERK1/2, and smoothmuscle $\alpha$-actin, which is a marker of myofibroblasts (Simpson et al. 2009). Type III collagen has an important role in wound healing, because the expression of type III collagen increases early during wound healing processes (Liu et al. 1995). Human fibroblasts with mutations in the COL5A1 and COL3A1 genes do not organize collagens and fibronectin in the ECM, down-regulate $\alpha 2 \beta 1$ integrin, and recruit $\alpha v \beta 3$ instead of $\alpha 5 \beta 1$ integrin (Zoppi et al. 2004). The $\alpha v \beta 3$ receptor (vitronectin receptor) and the $\alpha 5 \beta 1$ receptor (fibronectin receptor) have been shown to be important in myofibroblast differentiation (Lygoe et al. 2007). Mice deficient in type III collagen have an increased myofibroblast density in the wound granulation tissue, as evidenced by the increased expression of the myofibroblast marker, smooth-muscle $\alpha$-actin, and wounds in such mice have significantly more scar tissue area compared with that of wild-type mice (Volk et al. 2011). In our study, HDF cultured on the low-dose ASESM environment induce a cytoskeletal rearrangement and change the expression of ECM, especially type III collagen, by affecting the interaction with integrin. We hypothesize that the newly designed PMBN-ASESM biointerface mimicks the microenvironment of both early and late remodeling in the wound healing process.

The inductive mechanism of these highly expressed genes (type III collagen, decorin, and MMP2) in HDF on the low dose of ASESM seems to be related to the cell shape, cellular environment, cell-cell interaction (low or high cell density), and ECM components or its structure. Because the high dose of ASESM does not activate these three genes, regardless of the cell density, we suggest that the particular ECM environment that is mediated by the low ASESM dose plays a pivotal role for the observed cell adhesion mode and therefore affects the gene expression pattern. ASESM, the hydrolyzed material of ESM, might contain the ECM-related molecules and MMPs as regulatory factors. We hypothesize that HDF secrete exactly the same or similar ECM proteins to those of the surrounding ECM, because fibroblasts are known to be responsive to various signals (Discher et al. 2005). Cells express the corresponding genes in response to their surrounding environment and to environmental stimuli (mechanical force or UV stress, etc.). Cells and ECM interact with each other and adapt to their environment. If the environment of the cells is adequate, they can maintain the optimal condition for their survival. Mesenchymal stem cells retain their pluripotency providing that the surrounding environment remains suitable for stem cells.

In this study, the three genes (type III collagen, decorin, and MMP2) that are expressed in younger skin or early wound healing process are highly expressed by HDF on low doses of ASESM. This probably occurs because the required structure pre-exists or is assembled de novo on PMBN.

ESM might contain almost all ECMs and ECM-regulatory gene products that have been evolutionally conserved in avians. Previous studies have shown that ESMs have a fibrous network mainly comprised of type I, V, and X collagen, glucosamine, desmosin, hyaluronic acid (Ha et al. 2007; Osuoji 1971; Wong et al. 1984); because these molecules are cross-linked by lysyl oxidase (Harris et al. 1980), each component in ESMs cannot exist independently. In the low concentration solution (low-dose ASESM), hydrophilic small molecules that are produced by the moderately alkaline hydrolyzed ESM might be assembled in such a manner that a large amount of water surrounds it, thereby creating an ECM environment that mimicks younger skin. In contrast, the high concentration of ASESM (highdose ASESM) might induce the self-assembly of the ASESM components, such as the more hydrophobic ECM molecules (collagens) that relatively easily exclude water.

Such a self-assembly of macromolecules (ECM-related fibrils) might be involved during embryonic development and wound healing process in the animal body. By using a novel combination of a natural (ESM) and artificial cell membrane interface (PMBN), ECM components in ESM might be able to self-assemble differently and provide a distinct ECM environment depending on the ASESM concentration. Thus, the cells adhere to the respective environment and express the observed ECM components. The ASESM-PMBN system in this study should be 
applicable in various mechanistic studies of biological processes, such as wound healing, embryonic development, acquisition of tissue specificity, evolution and morphogenesis, at the molecular level.

In conclusion, ASESM has been stably conjugated to PMBN and provides an ECM environment for HDF. ESM also acts as a scaffold for eggshell mineralization and might have properties for capturing various factors and molecules that are necessary for avian embryonic development. Recently, a clinical evaluation of natural ESM has also reported joint and muscle pain relief (Ruff et al. 2009a, b). Although the immobilization of knee joints induces articular contracture associated with sequential changes of disordered ECM alignment, increased collagen glycation with pentosidine, and decreased cell numbers (Lee et al. 2010), the possible in vivo application of ASESM might not only stabilize connective tissues such as joints, but also stimulate ECM production therein. Further study of the ASESM-PMBN system with respect not only to skin wound healing, but also to the treatment of joint pain such as osteoarthritis should be considered, as MPC polymer has been shown to improve the surface grafting of artificial joints dramatically (Moro et al. 2004) and is under clinical trial awaiting biomedical use. In addition, our ASESM-PMBN system provides a rare experimental model for studying a mutually dynamic biological process in vitro: the cell responds to the surrounding microenvironment by secreting an ECM, and vice versa, the environment stimulates the cell.

Acknowledgements We appreciate the valuable comments of the reviewers. We thank the Radio Isotope Center and Center for NanoBio Integration (CNBI), The University of Tokyo for the use of analytical equipment, and we are grateful to Prof. Yoshihiko Nakamura at The University of Tokyo for administration of the "Cell to Body Dynamics Project", which included this ESM cooperative project.

Open Access This article is distributed under the terms of the Creative Commons Attribution Noncommercial License which permits any noncommercial use, distribution, and reproduction in any medium, provided the original author(s) and source are credited.

\section{References}

Ahlborn G, Sheldon BW (2005) Enzymatic and microbiological inhibitory activity in eggshell membranes as influenced by layer strains and age and storage variables. Poult Sci 84:1935-1941

Aiba-Kojima E, Tsuno NH, Inoue K, Matsumoto D, Shigeura T, Sato T, Suga H, Kato H, Nagase T, Gonda K, Koshima I, Takahashi K, Yoshimura K (2007) Characterization of wound drainage fluids as a source of soluble factors associated with wound healing: comparison with platelet-rich plasma and potential use in cell culture. Wound Repair Regen 15:511-520

Betz P, Nerlich A, Wilske J, Tubel J, Penning R, Eisenmenger W (1993) Analysis of the immunohistochemical localization of collagen type III and V for the time-estimation of human skin wounds. Int J Leg Med 105:329-332
Boraldi F, Croce MA, Quaglino D, Sammarco R, Carnevali E, Tiozzo R, Pasquali-Ronchetti I (2003) Cell-matrix interactions of in vitro human skin fibroblasts upon addition of hyaluronan. Tissue Cell $35: 37-45$

Bruckner P (2010) Suprastructures of extracellular matrices: paradigms of functions controlled by aggregates rather than molecules. Cell Tissue Res 339:7-18

Burley RW, Vadehra DV (1989) The avian egg, chemistry and biology. Wiley, New York

Corsi A, Xu T, Chen XD, Boyde A, Liang J, Mankani M, Sommer B, Iozzo RV, Eichstetter I, Robey PG, Bianco P, Young MF (2002) Phenotypic effects of biglycan deficiency are linked to collagen fibril abnormalities, are synergized by decorin deficiency, and mimic Ehlers-Danlos-like changes in bone and other connective tissues. J Bone Miner Res 17:1180-1189

Danielson KG, Baribault H, Holmes DF, Graham H, Kadler KE, Iozzo RV (1997) Targeted disruption of decorin leads to abnormal collagen fibril morphology and skin fragility. J Cell Biol 136:729-743

Debelle L, Tamburro AM (1999) Elastin: molecular description and function. Int J Biochem Cell Biol 31:261-272

Diegelmann RF, Evans MC (2004) Wound healing: an overview of acute, fibrotic and delayed healing. Front Biosci 9:283-289

Discher DE, Janmey P, Wang YL (2005) Tissue cells feel and respond to the stiffness of their substrate. Science 310:1139-1143

El Sherif A, Yano F, Mittal S, Filipi CJ (2006) Collagen metabolism and recurrent hiatal hernia: cause and effect? Hernia 10:511-520

Evanko SP, Angello JC, Wight TN (1999) Formation of hyaluronanand versican-rich pericellular matrix is required for proliferation and migration of vascular smooth muscle cells. Arterioscler Thromb Vasc Biol 19:1004-1013

Grzesiak JJ, Pierschbacher MD, Amodeo MF, Malaney TI, Glass JR (1997) Enhancement of cell interactions with collagen/glycosaminoglycan matrices by RGD derivatization. Biomaterials $18: 1625-1632$

Ha YW, Son MJ, Yun KS, Kim YS (2007) Relationship between eggshell strength and keratan sulfate of eggshell membranes. Comp Biochem Physiol A Mol Integr Physiol 147:11091115

Harris ED, Blount JE, Leach RM Jr (1980) Localization of lysyl oxidase in hen oviduct: implications in egg shell membrane formation and composition. Science 208:55-56

Ino T, Hattori M, Yoshida T, Hattori S, Yoshimura K, Takahashi K (2006) Improved physical and biochemical features of a collagen membrane by conjugating with soluble egg shell membrane protein. Biosci Biotechnol Biochem 70:865-873

Isnard N, Robert L, Renard G (2003) Effect of sulfated GAGs on the expression and activation of MMP-2 and MMP-9 in corneal and dermal explant cultures. Cell Biol Int 27:779-784

Itano N, Sawai T, Yoshida M, Lenas P, Yamada Y, Imagawa M, Shinomura T, Hamaguchi M, Yoshida Y, Ohnuki Y, Miyauchi S, Spicer AP, McDonald JA, Kimata K (1999) Three isoforms of mammalian hyaluronan synthases have distinct enzymatic properties. J Biol Chem 274:25085-25092

Jansen PL, Rosch R, Jansen M, Binnebosel M, Junge K, AlfonsoJaume A, Klinge U, Lovett DH, Mertens PR (2007) Regulation of MMP-2 gene transcription in dermal wounds. J Invest Dermatol 127:1762-1767

Jarvelainen H, Puolakkainen P, Pakkanen S, Brown EL, Hook M, Iozzo RV, Sage EH, Wight TN (2006) A role for decorin in cutaneous wound healing and angiogenesis. Wound Repair Regen 14:443-452

Kahari VM, Saarialho-Kere U (1997) Matrix metalloproteinases in skin. Exp Dermatol 6:199-213

Kanzler MH, Gorsulowsky DC, Swanson NA (1986) Basic mechanisms in the healing cutaneous wound. J Dermatol Surg Oncol 12:1156-1164 
Kerkela E, Saarialho-Kere U (2003) Matrix metalloproteinases in tumor progression: focus on basal and squamous cell skin cancer. Exp Dermatol 12:109-125

Kim SG, Akaike T, Sasagaw T, Atomi Y, Kurosawa H (2002) Gene expression of type I and type III collagen by mechanical stretch in anterior cruciate ligament cells. Cell Struct Funct 27:139-144

Konno T, Watanabe J, Ishihara K (2004) Conjugation of enzymes on polymer nanoparticles covered with phosphorylcholine groups. Biomacromolecules 5:342-347

Kontusaari S, Tromp G, Kuivaniemi H, Romanic AM, Prockop DJ (1990) A mutation in the gene for type III procollagen (COL3A1) in a family with aortic aneurysms. J Clin Invest $86: 1465-1473$

Kuivaniemi H, Tromp G, Bergfeld WF, Kay M, Helm TN (1995) Ehlers-Danlos syndrome type IV: a single base substitution of the last nucleotide of exon 34 in COL3A1 leads to exon skipping. J Invest Dermatol 105:352-356

Laurent TC, Fraser JR (1992) Hyaluronan. FASEB J 6:2397-2404

Lee S, Sakurai T, Ohsako M, Saura R, Hatta H, Atomi Y (2010) Tissue stiffness induced by prolonged immobilization of the rat knee joint and relevance of AGEs (pentosidine). Connect Tissue Res 51:467-477

Li F, Fan C, Cheng T, Jiang C, Zeng B (2009) Efficient inhibition of fibroblast proliferation and collagen expression by ERK2 siRNAs. Biochem Biophys Res Commun 382:259-263

Liu SH, Yang RS, al-Shaikh R, Lane JM (1995) Collagen in tendon, ligament, and bone healing. A current review. Clin Orthop Relat Res 318:265-278

Liu X, Wu H, Byrne M, Krane S, Jaenisch R (1997) Type III collagen is crucial for collagen I fibrillogenesis and for normal cardiovascular development. Proc Natl Acad Sci USA 94:1852-1856

Lygoe KA, Wall I, Stephens P, Lewis MP (2007) Role of vitronectin and fibronectin receptors in oral mucosal and dermal myofibroblast differentiation. Biol Cell 99:601-614

Meenakshi J, Vidyameenakshi S, Ananthram D, Ramakrishnan KM, Jayaraman V, Babu M (2009) Low decorin expression along with inherent activation of ERK1,2 in ear lobe keloids. Burns 35:519526

Mohan RR, Gupta R, Mehan MK, Cowden JW, Sinha S (2010) Decorin transfection suppresses profibrogenic genes and myofibroblast formation in human corneal fibroblasts. Exp Eye Res 91:238-245

Moro T, Takatori Y, Ishihara K, Konno T, Takigawa Y, Matsushita T, Chung UI, Nakamura K, Kawaguchi H (2004) Surface grafting of artificial joints with a biocompatible polymer for preventing periprosthetic osteolysis. Nat Mater 3:829-836

Mukhopadhyay A, Wong MY, Chan SY, Do DV, Khoo A, Ong CT, Cheong HH, Lim IJ, Phan TT (2010) Syndecan-2 and decorin: proteoglycans with a difference-implications in keloid pathogenesis. J Trauma 68:999-1008

Nomura Y (2006) Structural change in decorin with skin aging. Connect Tissue Res 47:249-255

Olsen BR (1995) The roles of collagen genes in skeletal development and morphogenesis. Experientia 51:194-195

Osuoji CI (1971) Acid glycosaminoglycan of eggshell membranes. Biochim Biophys Acta 244:481-483

Pickart L, Thaler MM (1973) Tripeptide in human serum which prolongs survival of normal liver cells and stimulates growth in neoplastic liver. Nat New Biol 243:85-87

Prockop DJ, Kivirikko KI (1984) Heritable diseases of collagen. N Engl J Med 311:376-386

Quaglino D Jr, Nanney LB, Kennedy R, Davidson JM (1990) Transforming growth factor-beta stimulates wound healing and modulates extracellular matrix gene expression in pig skin. I. Excisional wound model. Lab Invest 63:307-319
Rinn JL, Wang JK, Liu H, Montgomery K, Rijn M van de, Chang HY (2008) A systems biology approach to anatomic diversity of skin. J Invest Dermatol 128:776-782

Rose ML, Hincke MT (2009) Protein constituents of the eggshell: eggshell-specific matrix proteins. Cell Mol Life Sci 66:2707-2719

Rosenbloom J, Abrams WR, Mecham R (1993) Extracellular matrix 4: the elastic fiber. FASEB J 7:1208-1218

Ruff KJ, DeVore DP, Leu MD, Robinson MA (2009a) Eggshell membrane: a possible new natural therapeutic for joint and connective tissue disorders. Results from two open-label human clinical studies. Clin Interv Aging 4:235-240

Ruff KJ, Winkler A, Jackson RW, DeVore DP, Ritz BW (2009b) Eggshell membrane in the treatment of pain and stiffness from osteoarthritis of the knee: a randomized, multicenter, double-blind, placebo-controlled clinical study. Clin Rheumatol 28:907-914

Saarialho-Kere UK, Pentland AP, Birkedal-Hansen H, Parks WC, Welgus HG (1994) Distinct populations of basal keratinocytes express stromelysin-1 and stromelysin-2 in chronic wounds. J Clin Invest 94:79-88

Simeon A, Wegrowski Y, Bontemps Y, Maquart FX (2000) Expression of glycosaminoglycans and small proteoglycans in wounds: modulation by the tripeptide-copper complex glycyl-L-histidyl-L-lysine-Cu(2+). J Invest Dermatol 115:962-968

Simpson RM, Meran S, Thomas D, Stephens P, Bowen T, Steadman R, Phillips A (2009) Age-related changes in pericellular hyaluronan organization leads to impaired dermal fibroblast to myofibroblast differentiation. Am J Pathol 175:1915-1928

Stern R (2003) Devising a pathway for hyaluronan catabolism: are we there yet? Glycobiology 13:105R-115R

Takahashi K, Yamanaka S (2006) Induction of pluripotent stem cells from mouse embryonic and adult fibroblast cultures by defined factors. Cell 126:663-676

Tan EM, Hoffren J, Rouda S, Greenbaum S, Fox JWt, Moore JH Jr, Dodge GR (1993) Decorin, versican, and biglycan gene expression by keloid and normal dermal fibroblasts: differential regulation by basic fibroblast growth factor. Exp Cell Res 209:200-207

Tholozan FM, Gribbon C, Li Z, Goldberg MW, Prescott AR, McKie N, Quinlan RA (2007) FGF-2 release from the lens capsule by MMP-2 maintains lens epithelial cell viability. Mol Biol Cell 18:4222-4231

Tsang KY, Cheung MC, Chan D, Cheah KS (2010) The developmental roles of the extracellular matrix: beyond structure to regulation. Cell Tissue Res 339:93-110

Vitellaro-Zuccarello L, Garbelli R, Rossi VD (1992) Immunocytochemical localization of collagen types I, III, IV, and fibronectin in the human dermis. Modifications with ageing. Cell Tissue Res 268:505-511

Volk SW, Wang Y, Mauldin EA, Liechty KW, Adams SL (2011) Diminished type III collagen promotes myofibroblast differentiation and increases scar deposition in cutaneous wound healing. Cells Tissues Organs (in press)

von der Mark K, Park J, Bauer S, Schmuki P (2010) Nanoscale engineering of biomimetic surfaces: cues from the extracellular matrix. Cell Tissue Res 339:131-153

Wang JF, Olson ME, Reno CR, Kulyk W, Wright JB, Hart DA (2000) Molecular and cell biology of skin wound healing in a pig model. Connect Tissue Res 41:195-211

Watanabe J, Ishihara K (2007) Multiple protein-immobilized phospholipid polymer nanoparticles: effect of spacer length on residual enzymatic activity and molecular diagnosis. Nanobiotechnol 3:76-82

Welgus HG, Campbell EJ, Cury JD, Eisen AZ, Senior RM, Wilhelm SM, Goldberg GI (1990) Neutral metalloproteinases produced by human mononuclear phagocytes. Enzyme profile, regulation, and expression during cellular development. J Clin Invest 86:1496-1502 
Wilhelm SM, Collier IE, Kronberger A, Eisen AZ, Marmer BL, Grant GA, Bauer EA, Goldberg GI (1987) Human skin fibroblast stromelysin: structure, glycosylation, substrate specificity, and differential expression in normal and tumorigenic cells. Proc Natl Acad Sci USA 84:6725-6729

Wong M, Hendrix MJ, Mark K von der, Little C, Stern R (1984) Collagen in the egg shell membranes of the hen. Dev Biol 104:28-36

Wu JJ, Weis MA, Kim LS, Eyre DR (2010) Type III collagen, a fibril network modifier in articular cartilage. J Biol Chem 285:1853718544

Xu T, Bianco P, Fisher LW, Longenecker G, Smith E, Goldstein S, Bonadio J, Boskey A, Heegaard AM, Sommer B, Satomura K, Dominguez P, Zhao C, Kulkarni AB, Robey PG, Young MF
(1998) Targeted disruption of the biglycan gene leads to an osteoporosis-like phenotype in mice. Nat Genet 20:78-82

Zhang L, Tran N, Chen HQ, Kahn CJ, Marchal S, Groubatch F, Wang $\mathrm{X}$ (2008) Time-related changes in expression of collagen types I and III and of tenascin-C in rat bone mesenchymal stem cells under co-culture with ligament fibroblasts or uniaxial stretching. Cell Tissue Res 332:101-109

Zoppi N, Gardella R, De Paepe A, Barlati S, Colombi M (2004) Human fibroblasts with mutations in COL5A1 and COL3A1 genes do not organize collagens and fibronectin in the extracellular matrix, down-regulate alpha2beta1 integrin, and recruit alphavbeta3 instead of alpha5beta1 integrin. J Biol Chem 279:18157-18168 